\title{
Convergence Test of Model Reference Signal Adaptive SRM Drives
}

\author{
László Számel \\ BUDAPEST UNIVERSITY OF TECHNOLOGY AND ECONOMICS \\ H-1111 Budapest, Egry József u. 18. \\ Budapest, Hungary \\ Tel.: $+36 /(1)-463.29 .71$. \\ Fax: $+36 /(1)-463.36 .00$. \\ E-Mail:szamel@eik.bme.hu \\ URL: http://www.vgt.bme.hu/info/cv/szamellaszlo.htm
}

\section{Keywords}

«Adaptive control», «Control of Drive», «Non-linear control», «Servo-drive», «Switched reluctance drive»

\begin{abstract}
Nowadays switched reluctance motor (SRM) drives have been widely used in the field of controlled electric motor drives. The paper proposes a model reference signal adaptive control method for SRM drives. The main goal of the drive control is to improve dynamical performance by compensating for the motor nonlinearities. The adaptation practically works only in a relatively narrow speed error track (adaptation range) which is equal to approximately 20-100 revolution/min absolute value of speed error. The drawback of this limitation is the relatively short time for the algorithm to operate. At the same time the convergence of the algorithm is extremely fast which significantly reduces the effect of this drawback. Two more important advantages emerge when adaptation works only with small speed errors. First of all the controller at changing drive parameters adapts to parameters around the value specified by speed reference signal which also assists to speed the adaptation. The other significant positive effect is the disappearing of the problem coming from nonlinear systems that the response of the system can even differ in its character when the value, amplitude of the reference signal is changed. The convergence test of this control method was performed in an experimental drive system. A SRM of $6 / 8$ pole and $4 \mathrm{~kW}$ rated power was used. Simulation and experimental results are presented.
\end{abstract}

\section{Introduction}

In motion control systems are robustness against parameter changes and disturbance rejection of main interest. The model reference adaptive control has the following features:

- It makes the compliance of the system with varying operational conditions possible and ensures the behavior of the controlled system according to the prescribed reference model.

- It means such a special type of adaptive systems which results in nonlinear control systems. This is the reason why the analytical analysis is completed by Lyapunov stability criterium or by hyper-stability principle.

- Its planning and application is closely related to the using of computer methods.

- Simple realisation of the control algorithm.

In this paper the application of a model reference signal adaptive control to switched reluctance motors [1], [4], [6] is presented. 


\section{Drive system}

The block scheme of the examined drive system is shown in Fig.1. The supply unit consists of three main blocks, namely the RECTIFIER, the FILTER and the INVERTER. The inverter is a pulsed width modulated (PWM) one, marked with QP in the figure and it contains one-one switching transistor per phase and a brake chopper, not shown in the figure. The common point of phase windings is supplied by the PWM inverter. It is of autonomous operation and has an inner current control loop. The other ends of phase windings are connected to the phase switching transistors.

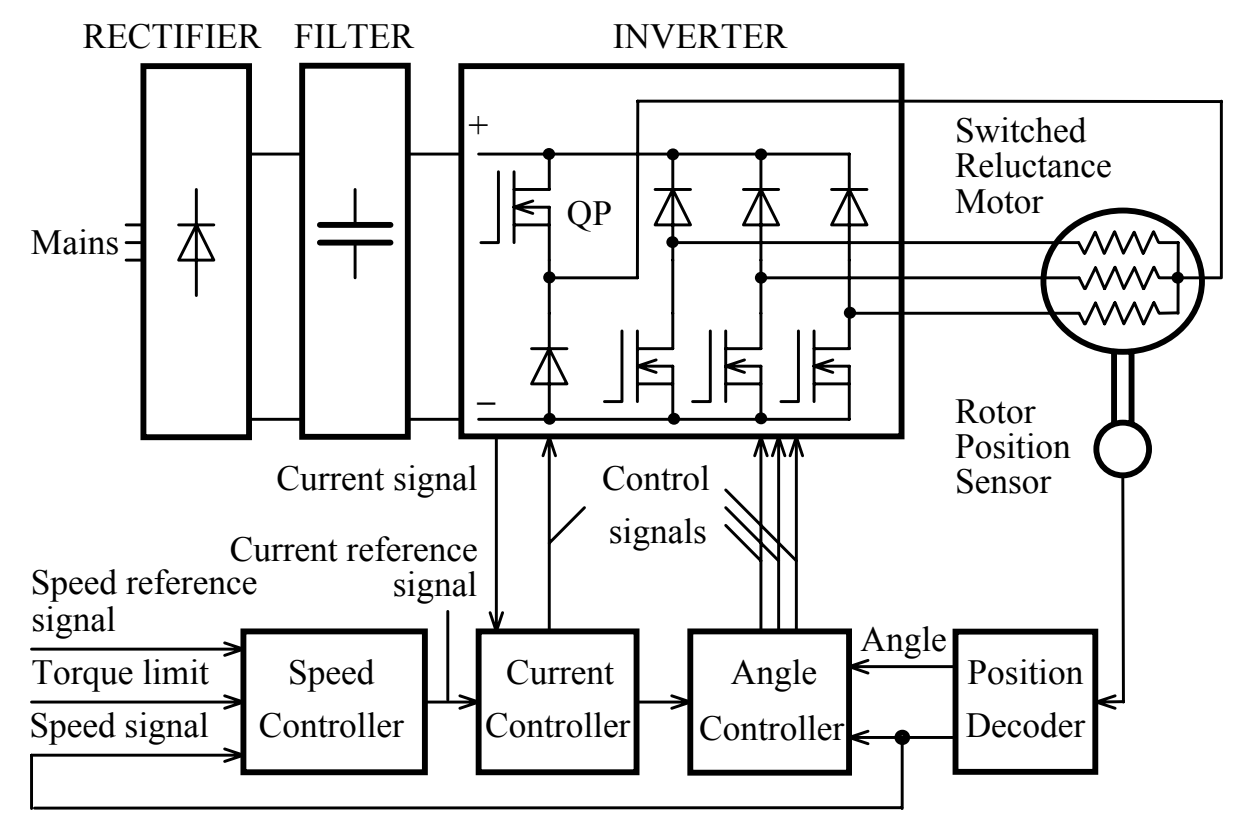

Fig. 1: Block scheme of drive system

It follows from the operation principle of SRM [1] [4] that its phase windings are to be excited at a well determined angle of rotor position in an appropriate order. This is why a Rotor Position Sensor is to be mounted on the shaft of the motor. In our case the position sensor is a resolver. It can be calculated from the pole numbers that the phase switchings have to follow each other by 15 degree. The resolver is supplied by an oscillator circuit, their signals are evaluated by a Position Decoder.

The Position Decoder has two outputs: the Angle and Speed signals. Based on the two signals, the Angle Controller composes the Control signals for the phase switching transistors.

Fundamentally, SRM drives have two control loops [1], the outer one is the speed loop, Speed Controller and the inner one is the current loop, Current Controller. The output signal of the Speed Controller serves for a Current reference signal of the Current Controller. The hardware and software tools together fulfil the two-loop control. The Current Controller produces the control signal for the PWM inverter, and receives the Current signal from the PWM inverter at the same time.

\section{Control of the SRM drive}

\section{Current control}

The Current Controller is totally based on its hardware solution. Based on the current reference signal, it controls the PWM inverter of fix frequency by installing an analog controller. The current feedback also comes from the PWM inverter. For the control of the sum of phase currents (Fig.1) it is suitable a simpler four-transistor inverter [4] and is not necessary a six-transistor one as in the case of control of phase currents independently from each other. But the detriment of the previous solution is that the torque pulsation can be decreased in a smaller degree by changing the turn-on and turn-off angles. 
Namely, in the case of the constant current reference signal the current increase is limited by the switched-off, but conducting phase current as the regulator controls the sum of two phase currents. The increase of the phase current at starting the conducting state can be forced by the modification of the current reference signal [7]:

$$
i_{r}=u \sum_{j=1}^{3} C_{j}+\sum_{j=1}^{3}\left(1-C_{j}\right) \cdot i_{j},
$$

where:

$i_{r} \quad$ is the current reference signal,

$i_{j} \quad$ is the current signal of phase $j$,

$u \quad$ is the output of the speed controller,

$C_{j} \quad$ is the control signal of phase $j$ (0 or 1 ).

The supplement of the first member of Eq. (1) makes the overlap of the phase conduction possible, while the effect of second member is to increase the reference signal with the current of the switchedoff, but not current-free phase.

\section{Speed control}

A model reference signal adaptive control is used for the speed control. Such an adaptive control has been succesfully elaborated by using a suitable chosen Lyapunov function to compensate the gain of the speed control loop [2], [8].

The Lyapunov function has been chosen in such a way that the model error should be decreased asymptotically and the gain of the speed control loop and the load should be compensated. In this case we have had to assume that the change of the loop gain and the load is smaller than the speed of the adaptation.

The arrangement of speed control loop is shown in Fig. 2 where an adaptation signal, signed by $g$ is added to the error signal. Applying the signal adaptation control, a $\mathrm{P}$ type controller of $K_{P}$ gain can ensure zero speed error as the adaptation signal $g$ can produce a current reference signal to compensate the loading current at zero speed error. The motor has been approximated by an integral element. The time constant of the closed current control loop has been neglected only in the interest of simpler adaptation algorithm.

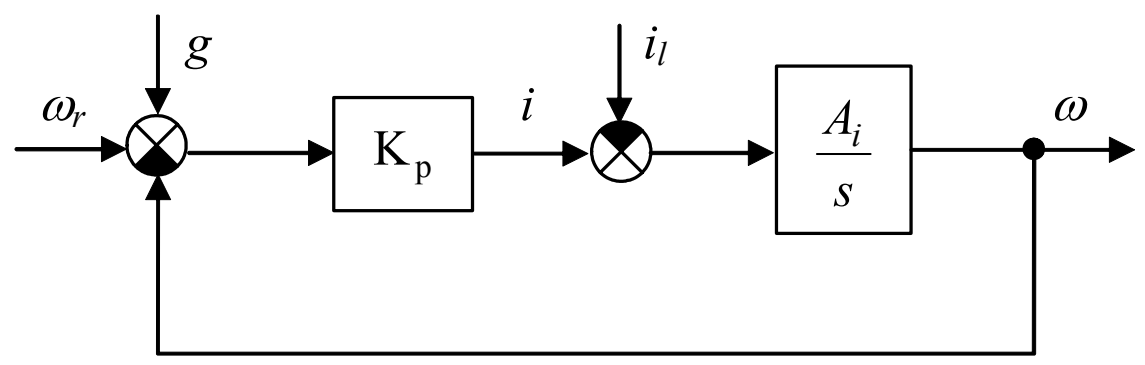

Fig.2: Block scheme of the speed control loop

The symbols of Fig. 2 are as follows:

$$
\begin{array}{ll}
A_{\mathrm{i}}=k_{\mathrm{m}} / J_{\mathrm{m}}, & \\
k_{\mathrm{m}} & \text { is the torque coefficient of the motor, } \\
J_{\mathrm{m}} & \text { is the total inertia related to the motor shaft, } \\
i & \text { is the current of the motor, } \\
i_{l} & \text { is the current equivalent to the load-torque, }
\end{array}
$$


$\begin{array}{ll}\omega & \text { is the speed, } \\ \omega_{r} & \text { is the speed reference signal. }\end{array}$

The relation between the acceleration current of dynamic torque and the speed can be written by the following transfer function:

$$
Y_{\omega\left(i-i_{l}\right)}=\frac{A_{i}}{S} .
$$

Regarding the block diagram of the motor the following differential equation is valid for the closed loop:

$$
\dot{\omega}+A_{i} K_{p} \omega=A_{i} K_{p}\left(\omega_{r}+g\right)-A_{i} i_{l} .
$$

The feature of the closed speed control loop has been taken into consideration by a parallel control model to be expressed by a one-storage proportional element. The differential equation of the first order system is:

$$
\dot{\omega}_{m}+q_{m} \omega_{m}=q_{m} \omega_{r},
$$

where the index $m$ refers to the model and $q_{m}$ is the reciprocal of the model time constant.

Using Eqs. (3) and (4) and introducing the expression $\varepsilon=\omega_{m}-\omega$ for the model error, the dynamic equation for the error is as follows:

$$
\dot{\varepsilon}+q_{m} \varepsilon=\left(q_{m}-A_{i} K_{p}\right)\left(\omega_{r}-\omega\right)+A_{i}\left(i_{l}-K_{p} g\right) .
$$

The adaptation signal $g(t)$ can be written in the following form:

$$
g(t)=g_{1}(\mathrm{t})\left(\omega_{r}-\omega\right)+g_{2}(t) .
$$

Substituting Eq. (6) for (5):

$$
\dot{\varepsilon}=-q_{m} \varepsilon+b_{1}\left(\omega_{r}-\omega\right)+b_{2},
$$

where

$$
\begin{aligned}
& b_{1}=q_{m}-A_{i} K_{p}\left(1+g_{1}(t)\right), \\
& b_{2}=A_{i}\left(i_{l}-K_{p} g_{2}(t)\right) .
\end{aligned}
$$

Let us compose the following Lyapunov function to produce the signal $g_{1}(t)$ and $g_{2}(t)$ :

$$
V=\frac{1}{2} \varepsilon^{2}+\frac{1}{2}\left(\beta_{1} b_{1}^{2}+\beta_{2} b_{2}^{2}\right),
$$

where $\beta_{1}$ and $\beta_{2}$ are positive constants.

The time-derivation of the Lyapunov function is:

$$
\dot{V}=\varepsilon \dot{\varepsilon}+\beta_{1} b_{1} \dot{b}_{1}+\beta_{2} b_{2} \dot{b}_{2} .
$$


Substituting Eq. (7) for (8):

$$
\dot{V}=-q_{m} \varepsilon^{2}+\left(\omega_{r}-\omega\right) b_{1} \varepsilon+b_{2} \varepsilon+\beta_{1} b_{1} \dot{b}_{1}+\beta_{2} b_{2} \dot{b}_{2} .
$$

If

$$
\dot{b}_{1}=-\left(\omega_{r}-\omega\right) \varepsilon / \beta_{1}
$$

and

$$
\dot{b}_{2}=-\varepsilon / \beta_{2},
$$

then

$$
\dot{V}=-q_{m} \varepsilon^{2}
$$

and it ensures the asymptotical stability of the model error. On the basis of Eqs. (7), (11) and by assuming that the variation of $A_{i}$ can be neglected compared to the speed of adaptation, the following adaptation algorithm is valid:

$$
\begin{aligned}
& \dot{g}_{1}(t)=\gamma_{1} \varepsilon\left(\omega_{r}-\omega\right), \\
& \dot{g}_{2}(t)=\gamma_{2} \varepsilon
\end{aligned}
$$

where $\gamma_{1}$ and $\gamma_{2}$ are positive constants, the free parameters of the adaptation. Taking the relations (6), (13) into consideration the following equation comes true:

$$
g(t)=\gamma_{1}\left(\omega_{r}-\omega\right) \int \varepsilon\left(\omega_{r}-\omega\right) d t+\gamma_{2} \int \varepsilon d t
$$

The block diagram of the control circuit introducing the adaptation signal $g(t)$ furthermore $g_{1}(t)=$ const., can be seen in Fig. 3. Taking the structure of control: it contains two parts. Reference signal which is led through a first order system and a PI controller with variable gain and integration time complemented with a filtered differentiating effect effecting only on the changing of the reference signal. Gain and differentiation time are also changing. The adaptation gain factor $\gamma_{2}$ gives the reciprocal of the integrating time constant of controller type PI, assuming $g_{1}(t)=0$.

The approaching block diagram of the adaptive control can be seen as extended version of the PF (integral element with separate proportional feedback loop, [3], [5], the input of the proportional element is only the speed) controller, so $\gamma_{2}$, one of the adaptation factors (which is the free parameter of the adaptive control) is given. Contraction of model-filtered reference signal and the PI controller can be transformed into a so-called PF controller when integration time of PI controller equals to the time constant of the model.

It follows from the determination of the Lyapunov function, that $b_{l}(t)$ will be equal to zero at the end of the adaptation. Using Eq. (7) the following relation is valid:

$$
1+g_{1}(t)=q_{m} /\left(A_{i} K_{p}\right)
$$

It can be seen from relation (15) and the block diagram of the control circuit, that the task of adaptation signal $g_{1}(t)$ is to ensure the constant gain in the loop. For fulfilling the constant integrating 
time constant, it is preferable to substitute $\gamma_{2}$ by $\gamma_{2}\left(1+g_{1}(t)\right)$. In this case $\gamma_{2}$ means the reciprocal value of the controller integration time even when $g_{1}(t)$ is nonzero and at the same time determines the value of $\gamma_{2}$. In such a way the neglection of the time constant of current control loop can be compensated.

Basic structure of the speed controller is PF type which firstly provides overshootless with its structure. On the other hand it ensures the fast compensation of speed variation caused by jump in the motor load.

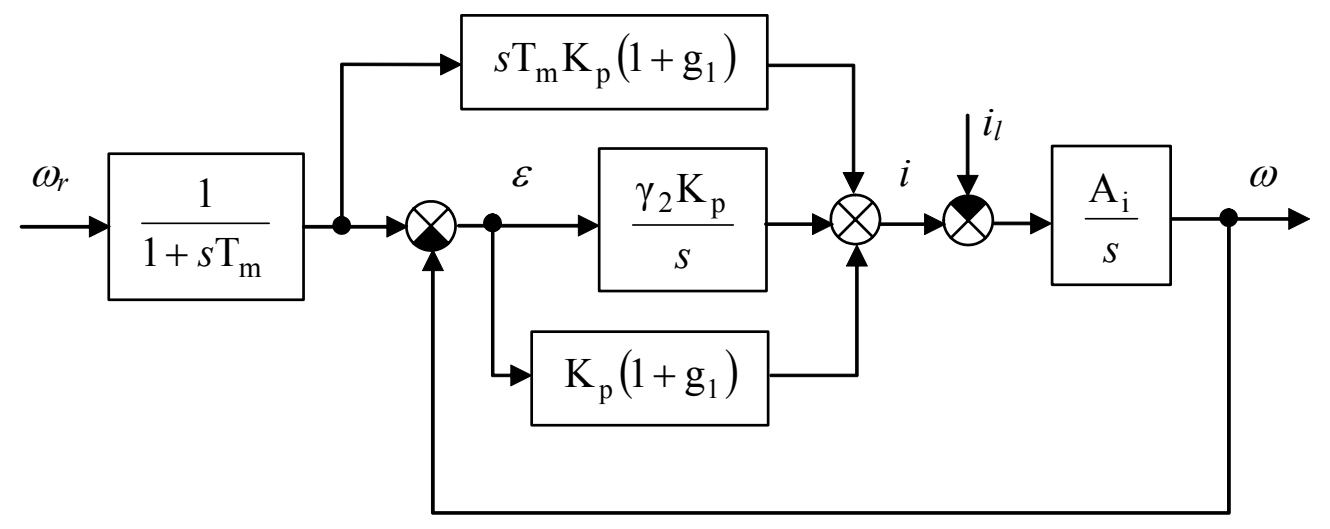

Fig.3: Block scheme of the adaptive speed control loop

In the case of sampled procedure the adaptation algorithm will be as follows:

$$
\begin{aligned}
& g[k]=g_{1}[k]\left(\omega_{r}[k]-\omega[k]\right)+g_{2}[k], \\
& g_{1}[k]=g_{1}[k-1]+\Gamma_{1} \varepsilon[k]\left(\omega_{r}[k]-\omega[k]\right), \\
& g_{2}[k]=g_{2}[k-1]+\Gamma_{2} \varepsilon[k]\left(1+g_{1}[k]\right) .
\end{aligned}
$$

It follows from the deduction that the asymptotical stability is valid if the model error does not change the sign. If the sign will change, the adaptation has to be stopped for one sampling step in general.

The control in recursive form supplemented with the adaptation algorithm will be as follows:

$$
\begin{aligned}
& u[k]=u[k-1]+K_{i} \varepsilon[k]\left(1+g_{1}[k]\right)+K_{P}\left(\left(1+g_{1}[k]\right) e[k]-\left(1+g_{1}[k-1]\right) e[k-1]\right), \\
& g_{1}[k]=g_{1}[k-1]+\Gamma_{1} \varepsilon[k]\left(\omega_{r}[k]-\omega[k]\right) .
\end{aligned}
$$

where
$u[k] \quad$ is the output of speed controller at the k-th sampling period,
$e[k] \quad$ is the error signal $\left(\omega_{r}[k]-\omega[k]\right)$,
$\varepsilon[k] \quad$ is the model error $\left(\omega_{m}[k]-\omega[k]\right)$,
$\omega[k] \quad$ is the speed feedback signal,
$\omega_{r}[k] \quad$ is the speed reference signal,
$g_{l}[k] \quad$ is the adaptation signal,
$[k-1] \quad$ is the previous sampling period,
$\Gamma_{1} \quad$ is a positive constant,
$K_{p}, K_{i} \quad$ are the proportional and the integral constants.

This control has been tested by the simulation program developed in the Department. First the adaptation has been examined without load and current limitation as the motor and the adaptation do contain non-linearities. In the interest of the adaptation stability the speed of change of the adaptation 
signal $g_{1}$ has to be limited. The signal $g_{1}$ can results in the significant oscillations without limitations as the change of the signal is possible in discrete times.

The current limitation can results in further problems. This limitation hinders the tracking of the model, to the effect of the above the signal $g_{1}$ will be too large or it can change in the reverse direction. For the elimination of the above problem the signal $g_{1}$ is not to be changed in the period of the limitation.

The digital output signal determined by the software is converted by a $\mathrm{D} / \mathrm{A}$ converter to an analog reference signal. The sampling time of speed loop is about $3 \mathrm{~ms}$. The speed feedback signal is determined by calculating the difference between the actual position value and the previous one of the resolver to digital converter. The measuring period ( $3 \mathrm{~ms}$ ) ensures an accuracy of $\pm 5 \mathrm{rpm}$.

\section{Convergence test}

To analyze the convergence of the signal adaptive speed control a square reference signal with $0.2 \mathrm{sec}$ period time has been applied.

Fig. 4 shows the characteristic $\left(1+g_{l}\right)$ time function of the convergence with $\mathrm{K}_{\mathrm{p}}=1, \Gamma_{1}=0,0001$ and $\Gamma_{2}=0,2$. Speed reference signal changes from 1000 to $15001 / \mathrm{min}$ and back in every $0.1 \mathrm{sec}$.

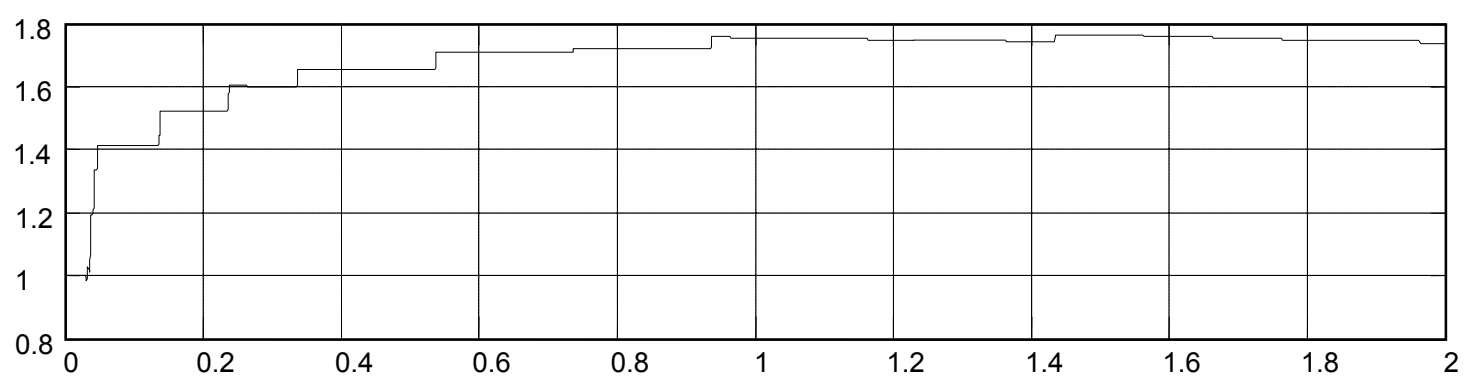

Fig.4: Convergence of $\left(1+g_{l}\right)$ adaptation signal: $\Gamma_{1}=0,0001$, ref. signal is $1000-15001 / \mathrm{min}$

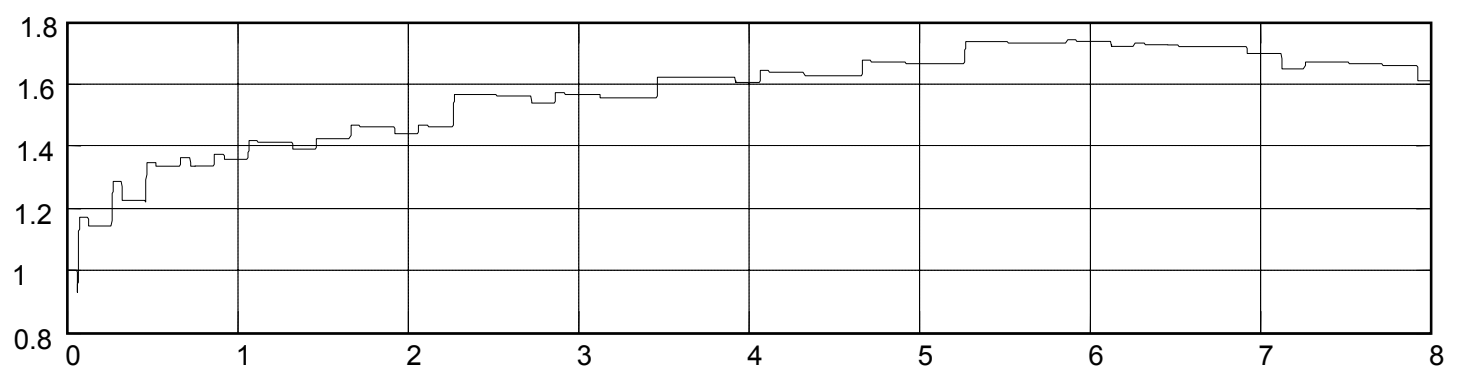

Fig.5: Convergence of $\left(1+g_{l}\right)$ adaptation signal: $\Gamma_{1}=0,0001$, ref. signal is $1000-20001 / \mathrm{min}$

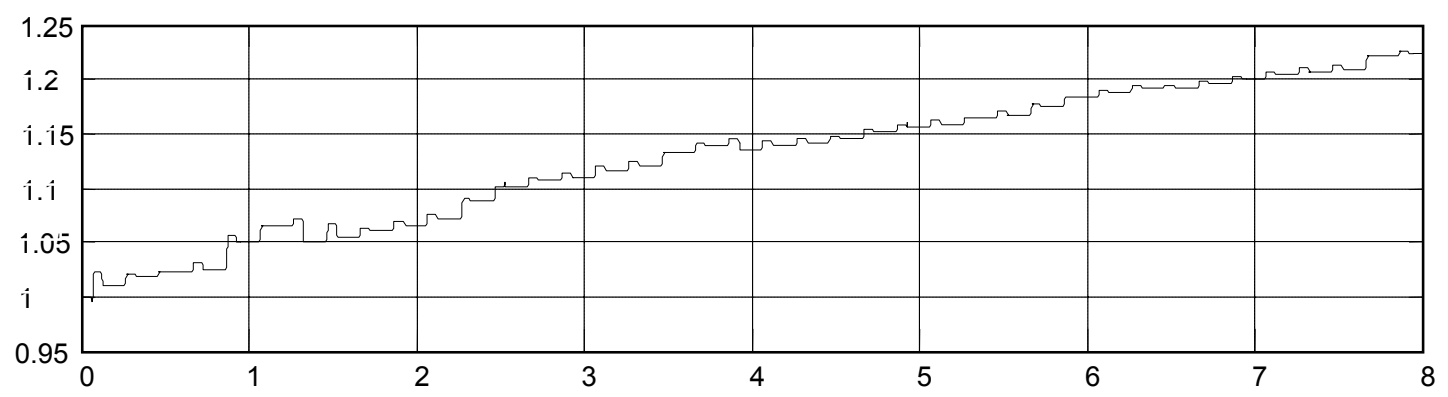

Fig.6: Convergence of $\left(1+g_{1}\right)$ adaptation signal: $\Gamma_{1}=0,00001$, ref. signal is $1000-20001 / \mathrm{min}$ 


\section{Summary for the simulation results of model reference signal adaptive control}

According to the simulation investigations convergence of the model reference signal adaptive control at switched reluctance drives with significant torque ripples can be ensured with the next conditions:

- Input of the first-order reference model only determines its output when the filtered current reference signal is lower with a given $\Delta \mathrm{I}$ value (in the simulation it has been set to $1 \mathrm{~A}$ ) than its limit otherwise output is identical to the speed feedback signal. So the model works like a first-order proportional lag element only in operation without current limitations.

- Adaptation is executed when two conditions are true at the same time:

- Filtered current reference signal is lower with a given $\Delta \mathrm{I}$ value (in the simulation it has been set to $1 \mathrm{~A}$ ) than current limit and

- Absolute value of the speed error signal is higher than a given $\Delta \mathrm{n}$ value (in the simulation it has been set to 20 revolution/min).

- The appropriate selection of $\Gamma_{1}$ adaptation factor also has important effect on the sufficient convergence.

Because of the restrictions described in point 2. adaptation practically works only in a relatively narrow speed error track (adaptation range) which is equal to approximately 20-100 revolution $/ \mathrm{min}$ absolute value of speed error. The drawback of this limitation is the relatively short time for the algorithm to operate. At the same time the convergence of the algorithm is extremely fast which significantly reduces the effect of this drawback. Two more important advantages emerge when adaptation works only with small speed errors. First of all the controller at changing drive parameters adapts to parameters around the value specified by speed reference signal which also assists to speed the adaptation. The other significant positive effect is the disappearing of the problem coming from nonlinear systems that the response of the system can even differ in its character when the value, amplitude of the reference signal is changed.

\section{Ripple reduced control method}

The ripple free operation of the SRM drives can be realize only with an current waveform depending on the angle, speed and torque [6]. The proposed ripple reduced method changes only the turn-on and the turn-off angle in function of the speed and current reference. The optimum turn-on and turn-off angles of the SRM drive has been determined by computer simulation based on the measured results of the analysed drive. The optimum solution has been fulfilled by four cycles embedded into each other. Two outer cycles give the current and speed reference signals, while two inner ones provide the turn-on and turn-off angles. By this one-one optimum angle pair can be determined to all operating points.

It can be considered an interesting result that the criterion of the minimum torque pulsation does not provide an optimum solution in all cases. The torque pulsation will be minimum in the speed-current plane only in case if the torque of the motor is relatively small. For this reason a good result can be achieved in a way if the relative, i.e. compared to the torque of motor, torque pulsation is minimized.

The angle control of the drive determines the actual turn-on and turn-off angles with a two-variable interpolation from the results stored in a look-up table and calculated by the above method.

\section{Results}

In Fig. 7 one of many executed simulations is shown. Fig. 7 shows the run-up with speed controller with model reference signal adaptive control (Eq. (16), Eq. (17)) with turn-on and turn-off angles depending on the speed and current reference and with current reference compensation (Eq. (1)).

The tests were completed by the described drive system. The test results have supported our theoretical investigations. The loading machine was a DC motor. Its inertia is about a triple of that of SRM. Fig. 8 shows the speed and current curves in the course of starting without current reference 

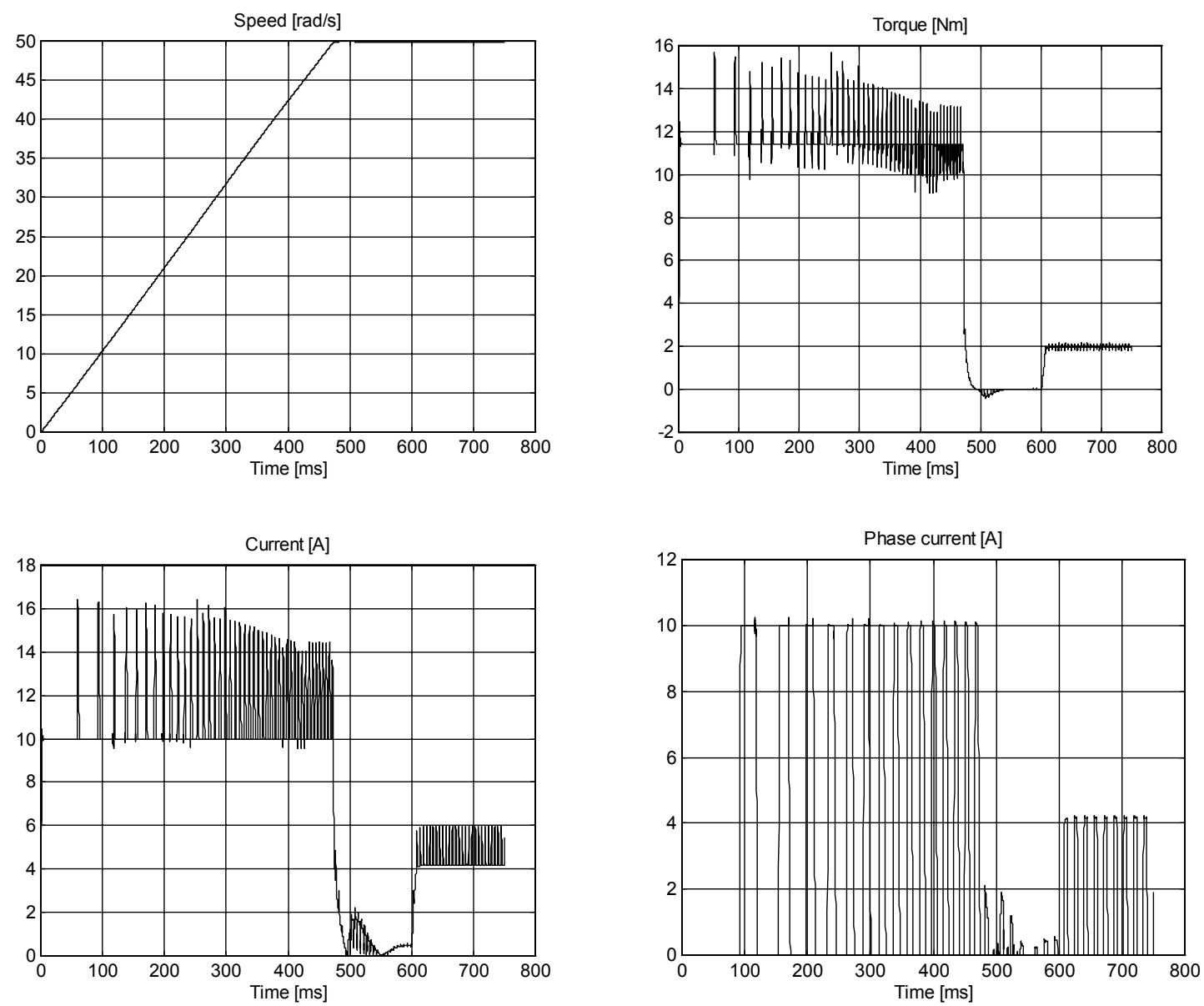

Fig. 7: Simulation results with model reference signal adaptive speed control

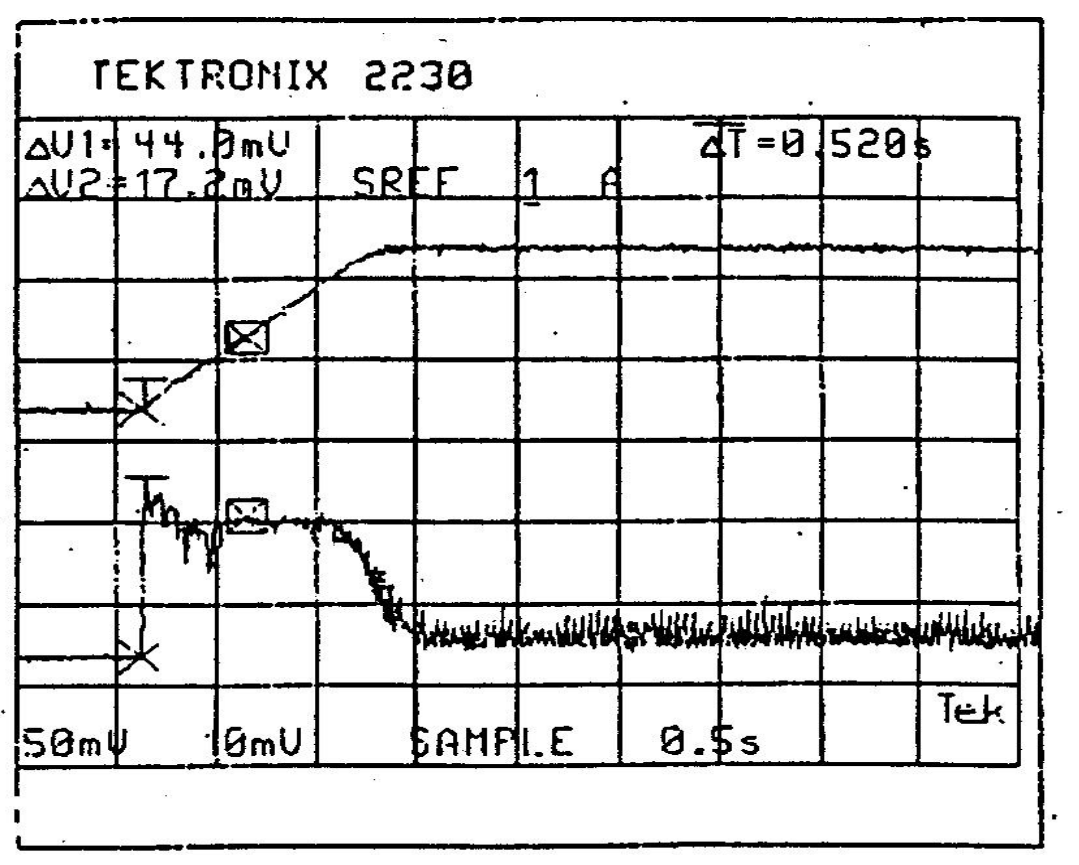

Fig. 8 Oscillogram of the speed and current 
compensation. The upper curve is the speed $(1500 \mathrm{rpm})$, the lower one is the current flowing in the common point of stator windings $(10 \mathrm{~A} / \mathrm{div})$. It is related to the no-load operation mode.

The experiences show that the model reference signal adaptive control suggested in this paper works without overshooting.

\section{Conclusion}

Basic structure of the speed controller is PF type (integral element with separate proportional feedback loop, [3], [5]) which firstly provides overshootless with its structure. On the other hand it ensures the fast compensation of speed variation caused by jump in the motor load. Thanks to the first-order model adaptation algorithm does not need acceleration measuring. The deducted algorithm is extremely fast and it can provide stability even at jumps in the load. With appropriate choice of the adaptation range convergence of the adaptation can even be ensured at SRM drives with significant torque ripples. Although normally because of the nonlinear characteristic of the adaptation, response of the system also depends on value of the jump in the reference signal this does not cause any problems in the proposed adaptive control as adaptation only happens in a narrow track of the reference signal named adaptation range. The wideness of the adaptation range is determined firstly by the torque ripples (with very low error adaptation does not work) and on the other hand the section where adaptation algorithm can work that is where limitations does not take effect depends on the agility of the speed controller (constituting time constant). At servo drives speed error belongs to it is about 50-100 revolution/min so it could be seen approximately independent from the jump magnitude in the reference signal. The experiences show that the model reference signal adaptive control suggested in this paper works without overshooting. Though this method requires a longer calculation period it is less sensible to the variations of parameters. The simulations and experimental results demonstrate that the proposed method is a promising tool to control the SRM drives.

\section{References}

[1]. Bose, B.K. - Miller, T.G.E.: Microcomputer Control of Switched Reluctance Motor. IEEE/IAS Annual Meeting. 1985. pp. 542-547.

[2] Cat, P.T. - Somlo, J.: On the Model Reference Adaptive Control of "n" Degree of Freedom Manipulators, MTA-SZTAKI. Working Paper, 1987. (No. E/48/87)

[3]. Diep, N.V. - Szamel, L.: Up-to-date Control Strategy in the Regulators of Robot Drives. PEMC'90, Budapest. 1990. pp. 811-815

[4]. Miller, T.J.E.: Brushless permanent-magnet reluctance motor drives. Oxford University Press. 1989

[5] Perdikaris, G.A. - VanPatten, K.W.: Computer Schemes for Modeling, Tuning and Control of DC Motor Drive Systems, PCI Proc., Mar.1982, pp. 83-96

[6]. Stankovic, Alexander. - Tadmor, Gilead.: On Torque Ripple Reduction in Current-Fed Switched Reluctance Motors. IEEE Transactions on Industrial Electronics, Vol. 46, No. 1, February 1999. pp. 177-183

[7]. Szamel, Laszlo.: Ripple reduced control of switched reluctance motor drives, EDPE2001, International Conference on Electrical Drives and Power Electronics, Podbanské (Slovak Republik), 3-5 October 2001. pp. 48-53

[8]. Szamel, Laszlo.: Model Reference Adaptive Control of SRM Drives, EPE-PEMC 2002, $10^{\text {th }}$ Int. Power Electronics and Motion Control Conf., Cavtat \& Dubrovnik (Croatia), 9-11 September 2002. pp. 466, CD-ROM: Paper No. T11-007 